\title{
émulations
}

\section{Laurent Dornel - Les étrangers dans la Grande Guerre}

\section{Pierre-Louis Buzzi}

Émulations - Revue de sciences sociales

2016, «Comptes rendus critiques, En ligne »

\section{Article disponible à l'adresse suivante}

https://ojs.uclouvain.be/index.php/emulations/article/view/7133

\section{Pour citer cet article}

Pierre-Louis Buzzi, « Laurent Dornel — Les étrangers dans la Grande Guerre », Émulations, en ligne. Mise en ligne le 10 mars 2016.

DOI : 10.14428/emulations.cr.017

Distribution électronique : Université catholique de Louvain (Belgique) : ojs.uclouvain.be

(C) Cet article est mis à disposition selon les termes de la Licence Creative Commons Attribution, Pas d'Utilisation Commerciale 4.0 International. http://creativecommons.org/licenses/by-nc/4.0/

Éditeur : Émulations - Revue de sciences sociales / Presses universitaires de Louvain https://ojs.uclouvain.be/index.php/emulations

ISSN électronique : 1784-5734

UCL PRESSES 


\title{
Laurent Dornel - Les étrangers dans la Grande Guerre
}

\begin{abstract}
Pierre-Louis Buzzi ${ }^{1}$
Recensé : Laurent Dornel, Les étrangers dans la Grande Guerre, Paris, La Documentation Française, 2014, 87 p.

Voilà un petit ouvrage de synthèse (moins d'une centaine de pages), issu d'une collection de vulgarisation sur l'histoire de l'immigration en France, qui constitue une introduction à une histoire oubliée de la Première Guerre mondiale : celle des étrangers, marquant ainsi le renouveau historiographique que peut connaître l'histoire du premier conflit mondial, dont on pourrait croire, à l'heure du Centenaire, que tout a été dit.

Maitre de conférences en histoire contemporaine à l'Université de Pau, Laurent Dornel est l'un des spécialistes de la question migratoire en France au XIX ${ }^{e}$ siècle et début XXe. Sa thèse, parue en 2004 sous le titre La France hostile chez Hachette Littérature, est désormais un livre de référence sur le sort et l'accueil des immigrés en France entre 1870 et 1914 . Avec cette nouvelle publication, Laurent Dornel prolonge son étude avec le premier conflit mondial sur lequel il avait déjà publié des articles passionnants (Dornel, 1995 et 2012).

Outre l'extension de la problématique, l'auteur étend son propos à toutes les catégories d'étrangers : les soldats appartenant à d'autres nations que la France mais qui viennent combattre en France ; les réfugiés issus de pays voisins et qui cherchent refuge en France; les immigrés, ces étrangers déjà présents en France avant le conflit et dont la situation et le statut varie en fonction de la situation du pays d'origine (pays neutres, alliés ou en guerre contre la France); les coloniaux; et les travailleurs étrangers que l'Etat français fait venir de divers pays, notamment européens pour travailler dans divers secteurs de l'économie, tant dans les champs que dans les usines de guerre.

Dans un premier chapitre, Laurent Dornel revient donc sur la diversité des situations et des statuts des étrangers en France. Y sont abordés le cas des étrangers issus des nations en guerre contre la France (45 000 étrangers sont internés au cours de la guerre), le cas particulier des Alsaciens-Lorrains qui doivent passer par des camps de triage, le sort des réfugiés et des évacués, en particulier celui des Belges dont 325000 sont recensés en novembre 1918, et celui des neutres, c'est-à-dire, des étrangers appartenant à une nation restée en dehors du conflit. La question des dénaturalisations est bien abordée
\end{abstract}

${ }^{1}$ Professeur certifié d'histoire-géographie en collège, titulaire d'un master de recherche en histoire contemporaine (Université de Strasbourg). 
et permet de rappeler que suite à la loi du 7 avril 1915, 25000 naturalisations sont révisées et aboutissent à 549 déchéances de nationalité et 8000 internements.

Au cours de la Première Guerre mondiale, des centaines de milliers d'étrangers et de coloniaux viennent combattre en France. L'auteur traite de ce sujet dans un second chapitre, abordant la question des 600000 combattants indigènes mobilisés parmi les troupes coloniales, dont 134000 Noirs et 158000 Algériens musulmans mobilisés qui rejoignirent l'Europe, et 20 à 25000 tirailleurs noirs qui servirent en France. L. Dornel approfondit ce point dans plusieurs sections consacrées aux différentes formes de recrutement. La conscription est ainsi rendue obligatoire en Algérie par deux décrets de septembre 1916. Au total, 40000 hommes furent appelés et mobilisés cette année, mais ces recrutements massifs ne se firent pas sans susciter de vives réactions, voire des révoltes, chez ces populations. Les conséquences pour ces soldats sont grandes, « outre l'expérience combattante, il y eut l'arrachement à la terre natale et le départ vers une métropole le plus souvent totalement inconnue » (p. 24). Il est vrai que pour un grand nombre d'entre eux, l'acclimatation fut difficile, c'est pourquoi dès la fin de 1914, les soldats noirs furent envoyés « en hivernage » dans des camps du midi de la France, et fut adopté le principe de ne les envoyer au combat qu'entre avril et octobre. Laurent Dornel insiste longuement sur leur condition en France. Si très peu d'entre eux bénéficient de promotion, « les soldats coloniaux furent l'objet d'une réelle attention de la part des autorités françaises (...). Le commandement fut confié à des officiers connaissant les coutumes et les langues du pays d'origine ; les fêtes traditionnelles et les prescriptions alimentaires furent respectées, tout comme les rites d'inhumation islamiques » (p. 26). On peut regretter la brièveté du paragraphe concernant les volontaires étrangers et la Légion étrangère sur laquelle il y a tant à dire, que ce soit pour les Italiens engagés dans la Légion Garibaldienne notamment étudiés par P. Milza (1986), H. Heyriès (2005), et S. Prézioso (2010), les Tchèques étudiés par J.-P. Namont (2004), les Juifs russo-polonais étudiés par P. Landau (1999) et bien d'autres. Par ailleurs, la question des fils d'étrangers ou des naturalisés dans l'armée française n'est pas abordée, au contraire des armées ennemies et alliées que l'auteur aborde sur plusieurs pages.

Dans un troisième chapitre, l'auteur approfondit la question des « étrangers et coloniaux au travail » (p. 34), c'est-à-dire de la mobilisation économique des étrangers dans les usines et les champs en France, rappelant que ces travailleurs venaient surtout d'Algérie (près de 80 000), du Maroc (35 000), de Tunisie (18 500), d'Indochine (49 000), de Chine ( 37000$)$, de Grèce (25 000), d'Italie (20 000), du Portugal (15 à 23000 selon les auteurs) et d'Espagne (10 000). Mais ces chiffres, prévient avec raison l'auteur, « ne tiennent compte ni des travailleurs qui se déplaçaient librement et/ou qui n'étaient pas déclarés, ni des dizaines de milliers de réfugiés belges » (p. 36), auxquels on peut aussi ajouter les immigrés déjà présents en France avant 1914. Le propos de l'auteur est par ailleurs illustré par des photographies d'ouvriers étrangers au travail ou au cantonnement particulièrement intéressantes qui viennent donner un peu de relief à l'ouvrage. Ces travailleurs étrangers se voient obligés de détenir une « carte d'identité » à partir 
d'avril 1917 (en réalité plus un carnet qu'une carte), et qui constitue une étape importante dans l'histoire de l'identification des étrangers en France. Laurent Dornel insiste ensuite sur les structures qui permettent ce recrutement massif de travailleurs étrangers, et la mise en place d'une administration spécifique. C'est la première fois que l'immigration est entièrement prise en charge par l'État. L'auteur, qui avait déjà publié un article sur cette question (Dornel, 1995), souligne l'instauration d'une politique racialiste : « à chaque "race" correspondaient des aptitudes physiques et psychologiques particulières qui déterminaient des types d'emplois particuliers » (p. 46).

Dans le quatrième chapitre, L. Dornel aborde les contacts entre les populations françaises et les étrangers. Entre manifestations de xénophobie et mariages mixtes, les relations sont particulièrement diversifiées. Toutefois, contrairement à ce qu'affirme l'auteur, les statistiques sur les mariages entre une Française et un étranger ne sont pas rares (p. 59), puisqu'une enquête est lancée dans chaque département en 1919 (Buzzi, 2015). Il rappelle cependant que cette question des mariages mixtes a dès le début divisé à la fois les autorités politiques et militaires et les spécialistes des questions migratoires. Si l'on admet le mariage de Françaises avec des Européens, on est beaucoup plus réticent envers des « travailleurs dont les mœurs, la religion et les habitudes sociales sont trop éloignées de celles des Européens » (p. 60). Les enfants nés de ces unions sont alors considérés comme une menace pour l'ordre colonial. Il y aurait toutefois eu une quinzaine de mariages entre ouvriers chinois et femmes françaises, et cent-vingt Nord-Africains se seraient mariés au cours de la guerre en France (p. 63). Pendant de la surveillance de la vie sexuelle des coloniaux, la surveillance de leur correspondance est également particulièrement attentive: les photographies représentant des femmes françaises nues ou des cartes postales légères sont interceptées pour éviter de " porter atteinte à l'image de la femme blanche et donc conduire à la subversion des hiérarchies raciales et sexuelles dans l'empire » (p. 64). Laurent Dornel revient ensuite sur les tensions et les « violences raciales » qui prennent place dans le cadre de la Première Guerre mondiale, en rappelant que si dans un premier temps, les ouvriers français accueillirent relativement favorablement les étrangers et les coloniaux, très rapidement, les oppositions se firent de plus en plus nombreuses. Par exemple dès 1915, Léon Jouhaux, secrétaire confédéral de la CGT, s'opposa au recrutement des Chinois. L'auteur estime que du côté des ouvriers, «en dépit de quelques mouvements comme celui des garçons de café parisiens contre la main-d'œuvre espagnole en 1917, l'hostilité sembla globalement épargner les travailleurs étrangers et se tourner essentiellement contre les coloniaux et les Chinois » (p. 65). C'est là, à notre avis, une affirmation un peu rapide. Nos propres recherches sur les immigrés italiens pendant la guerre ont révélé bien des formes d'hostilités contre l'emploi d'ouvriers étrangers (Buzzi, 2015). Les travaux sur la question étant encore peu nombreux, on pardonnera à l'auteur, son affirmation un peu rapide. Laurent Dornel n'oublie pourtant pas d'aborder les affrontements xénophobes, mais il les limite aux coloniaux et Chinois, ce qui est bien dommage. Ainsi évoque-t-il l'une des premières rixes contre ces derniers au Creusot en novembre 1916, ou encore 
les rixes entre coloniaux : « les Indochinois, en raison de leur plus grand succès auprès du personnel féminin des établissements où ils travaillaient, eurent bien des soucis avec les Noirs et surtout les Nord-Africains » (p. 66).

Un dernier chapitre sur les étrangers en France dans l'après-guerre évoque la multiplicité des questions migratoires au sortir de la guerre. Si les troupes coloniales sont à l'honneur sur les Champs-Elysées lors du défilé du 14 juillet 1919, très vite l'attention est portée sur les problèmes de main-d'œuvre à laquelle la France est confrontée. L. Dornel rappelle ainsi que pendant le conflit, le gouvernement s'interroge sur les potentialités de main-d'œuvre étrangère pour l'après-guerre. La France a besoin de bras pour se reconstruire, tant économiquement que démographiquement. En ce qui concerne les coloniaux présents en France à la fin de la guerre, les autorités rencontrèrent des difficultés à les renvoyer : des coloniaux avaient réussi à échapper à la surveillance, d'autres voulaient rester en France, sans parler des complications techniques de rapatriement. Mais là aussi, l'attention de l'auteur reste principalement concentrée sur les coloniaux et non sur les immigrés européens, et notamment italiens qui participent comme entrepreneurs ou comme ouvriers à la Reconstruction des "Régions libérées ».

Pour conclure, la lecture de l'ouvrage très synthétique de Laurent Dornel nous laisse quelques regrets. Tout d'abord, au lieu de se concentrer sur une catégorie d'étranger, l'auteur brasse tous les types d'étrangers : les immigrés, les travailleurs coloniaux et les Chinois, les prisonniers étrangers, les soldats alliés ou ennemis, le cas des AlsaciensLorrains. La première partie de l'ouvrage fait un peu penser à un catalogue dont les différentes composantes semblent mal reliées les unes aux autres. D'autre part, ces différentes catégories d'étrangers n'ont pas toutes le droit au même traitement. Il est en effet regrettable de voir que l'auteur porte essentiellement son regard sur les coloniaux, pour lesquels le lecteur se réjouira de développements particulièrement intéressants, mais ceci au détriment des immigrés. Ces étrangers, présents dans la métropole avant la guerre et qui restent en France pendant le conflit, tels les Italiens ou les Belges, ne sont en effet que très peu évoqués. La faute ne peut être entièrement mise sur les épaules de l'auteur, car l'histoire des minorités pendant la Première Guerre mondiale reste un parent pauvre de l'historiographie française du premier conflit mondial. Toutefois, ce pan n'est pas pour autant vierge. Nous regrettons ainsi que la bibliographie, au-delà de sa brièveté, nécessaire pour le format du volume, soit presque exclusivement tournée vers les Chinois et les colonisés, oubliant ainsi les travaux de P. Landau sur les juifs russes, de M. Corriol sur les réfugiés serbes, de P. Namont (2004) sur la colonie Tchécoslovaque, d'E. Filhol (2004) sur les Tsiganes, de J.-N. Grandhomme (2008) sur les Alsaciens, d'U. Leberle (2000), H. Heyriès (2005), S Prézioso (2010), et C. Douki (2002) sur les Italiens, et bien d'autres encore (S. Kronenberger, 2014). Ainsi, si la brièveté des propos et des paragraphes peut aisément s'expliquer par les obligations éditoriales et l'objectif de vulgarisation, tout à fait noble, le déséquilibre entre les différentes catégories d'étrangers, en particulier pour les immigrés, est plus fâcheux. 


\section{Bibliographie}

Buzzi P.-L. (2015), Les immigrés italiens en Lorraine française pendant la Première Guerre mondiale (1914-1918), mémoire de master, Strasbourg, Université de Strasbourg.

CoRriol M. (1993), Les réfugiés serbes pendant la Première Guerre mondiale dans les Basses-Alpes, mémoire de maîtrise, Marseille, Université d’Aix-Marseille, 116 p.

DORNEL L. (2012), «Les travailleurs chinois vus par l'administration militaire française (1914-1918): assignation, identification et représentations», in Li MA (dir), Les travailleurs chinois dans la Première Guerre mondiale, Paris, CNRS Éditions, p. 265-284.

DORNEL L. (1995), «Les usages du racialisme. Le cas de la main-d'œuvre coloniale en France pendant la Première Guerre mondiale », Genèses, vol. 20, p.48-72.

Douki C. (2002), «Les émigrés face à la mobilisation militaire de l’Italie », 14-18 Aujourd'hui, vol. 5, p. 158-181.

FiLHOL E. (2004), Un camp de concentration français. Les Tsiganes alsaciens-lorrains à Crest 1915-1919, Grenoble, Presses Universitaire de Grenoble, 181 p.

Grandhomme J.-N. (2008), Boches ou Tricolores. Les Alsaciens-Lorrains dans la Grande Guerre, Strasbourg, La Nuée Bleue, 463 p.

HeYRIÈs H., MuRACCIOLE J.-F. (dir.) (2007), Le soldat volontaire en Europe au XX $\mathrm{X}^{\mathrm{e}}$ siècle. De l'engagement politique à l'engagement professionnel. Actes du colloque international de Montpellier du 3 au 5 avril 2003, Montpellier, PUM, 483 p.

HeYRIES H. (2005), Les Garibaldiens de 14. Splendeurs et misères des Chemises Rouges en France de la Grande Guerre à la Seconde Guerre mondiale, Nice, Serre Editeur, $672 \mathrm{p}$.

KRonenberger S. (2014), Des temps de paix aux temps de guerre : les parcours des travailleurs étrangers de l'Est et du Sud-Est de la France (1871-1918), thèse de doctorat, Nice, Université de Nice Sophia-Antipolis, 2 vol., 1049 p.

LANDAU P. (1999), Les Juifs de France et la Grande Guerre. Un patriotisme républicain, 1914-1941, Paris, CNRS éditions, 294 p.

LEBERLE U. (2000), Les immigrés italiens de Marseille pendant la Première Guerre mondiale, mémoire de maîtrise, Marseille, Université d’Aix-Marseille I, 147 p.

PREzioso S. (2010), «Les Italiens en France au prisme de l'engagement volontaire : les raisons de l'enrôlement dans la Grande Guerre (1914-1915)», Cahiers de la Méditerranée, vol. 81, p. 147-163. 
NAMONT J.-P. (2004), « La colonie tchécoslovaque en France pendant la Première Guerre mondiale. », Guerres mondiales et conflits contemporains, vol. 216, p. 41-57. 